\title{
AGLOMERAÇÃO E VALOR DE COMPRA EM CENTROS DE CONSUMO DE BAIXA RENDA
}

\author{
CROWDING AND SHOPPING VALUE IN LOW- \\ INCOME RETAIL CENTERS
}

Recebido 17/08/2012 Aceito $17 / 08 / 2013$

Izabelle Quezado ${ }^{1}$ Rômulo Bernardino Lopes da Costa² Verónica Lidia Peñaloza Fuentes ${ }^{3}$

\section{RESUMO}

Diante do crescente potencial de consumo das camadas de baixa renda, esta pesquisa se baseia nas escalas de Babin, Darden e Griffin (1994) e de Machleit, Kellaris e Eroglu (1994) com o objetivo de investigar o comportamento de compra hedônico e utilitário relacionado à percepção de crowding (aglomeração) do consumidor de baixa renda em dois centros de consumo. A partir da pesquisa quantitativa, realizada com 404 consumidores em ambiente real de varejo, os resultados revelaram que os indivíduos dos dois locais mostraram-se propensos ao comportamento utilitário e demonstraram sentir-se mais incomodados pela aglomeração no centro de consumo com menor infraestrutura. Houve indícios de que esse incômodo foi menos intenso em consumidores hedônicos.

Palavras-Chave: Consumo Hedônico, Consumo Utilitário, Crowding, Baixa Renda

\footnotetext{
1 Possui graduação em Comunicação Social - Publicidade e Propaganda pela Universidade de Fortaleza - UNIFOR e mestrado em Administração pela Universidade Estadual do Ceará - UECE. Atualmente é doutoranda em Administração de Empresas pela Universidade de Fortaleza - UNIFOR. Fortaleza, Ceará, Brasil. E-mail: izabellequezado@gmail.com.

2 Possui graduação em Ciências Contábeis pela Universidade Federal do Ceará - UFC. Atualmente é mestrando em Administração pela Universidade Estadual do Ceará - UECE. Fortaleza, Ceará, Brasil. E-mail: romuloblc@gmail.com.

${ }^{3}$ Possui graduação em Economia pela Universidad de Chile, mestrado e doutorado em Economia pela Universidade de São Paulo - USP e pós-doutorado em Psicologia Econômica pela Universidad de La Frontera. Fortaleza, Ceará, Brasil. E-mail: vero.pf@hotmail. com.
} 


\begin{abstract}
Considering the great potential of low-income consumption, this research is based on the scales developed by Babin, Darden and Griffin (1994) and by Machleit, Kellaris and Eroglu (1994), aiming to investigate hedonic and utilitarian consumer behavior and its relation with low-income perception of crowding. A research was performed with 404 consumers in real store environment and the results showed that consumers revealed being prone to utilitarian behavior in both centers. About crowding phenomenon, the consumers felt more uncomfortable by crowding at the commercial center with less infrastructure. There were indices that this discomfort was less intense in hedonic consumers.

Keywords: Hedonic Consumption, Utilitarian Consumption, Crowding, Low-income
\end{abstract}

\title{
1 INTRODUÇÃO
}

Até o final da década de 90, as classes sociais menos abastadas eram desconsideradas como integrantes do mercado consumidor (ZILBER; SILVA, 2010). Com o aumento do consumo no Brasil, devido principalmente à expansão do crédito, percebe-se um acréscimo relevante nas vendas e no fluxo de clientes nos centros de consumo de baixa renda (TORRES; BICHIR; CARPIM, 2006).

Há, no entanto, diversas definições do que seria a baixa renda, uma vez que cada país tem determinado nível de desenvolvimento, normas sociais e valores. No Brasil, o Instituto Brasileiro de Geografia e Estatística (IBGE, 2010) divide a população brasileira em cinco faixas de renda, com base no número de salários mínimos recebidos. A população com renda familiar acima de 20 salários mínimos pertence à classe $A$. Na classe $B$, estão inseridas as famílias com renda entre 10 e 20 salários mínimos. A classe $C$ é composta por indivíduos com renda familiar entre 4 e 10 salários mínimos. As classes $D$ e $E$ englobam as famílias com renda entre 2 e 4 salários mínimos. Para Prahalad (2005), a população de baixa renda do Brasil é a que pertence às classes C, D e E e que detém uma renda familiar mensal de até 10 salários mínimos, definição tomada como base nesta pesquisa.

Sob uma perspectiva sociocultural, Rocha e Silva (2010) acreditam que, para a população de baixa renda, o consumo é uma forma de inclusão social, não somente por suprir necessidades no que diz respeito ao valor utilitário de compra, mas, sobretudo, por possibilitar o acesso ao universo hedônico, motivado pela emoção e pelas fantasias do consumidor. Para Holbrook e Hirschman (1982), a compra hedônica está relacionada a aspectos multisensoriais, sendo a decisão de compra apenas um componente dentre todos os envolvidos na experiência de consumo. Em ambiente de varejo, o comportamento hedônico de consumo está relacionado positivamente, dentre outros fatores, à variedade de produtos (BELLENGER, 1977 apud HERNANDEZ, 2009). O comportamento de compra utilitário, por sua vez, está estritamente relacionado à racionalidade, uma vez que é caracterizado pela procura do menor preço e pela utilidade que um produto ou serviço tem para o consumidor (ANDERSSON; ENGELBERG, 2006). Esse tipo de consumo é reflexo de uma compra eficiente, por uma tarefa orientada (BABIN; DARDEN; GRIFFIN, 1994).

Esses valores de compra estão fortemente ligados a aspectos culturais. Babin, Darden e Griffin (1994) sugerem que os consumidores de países desenvolvidos têm uma maior tendência ao comportamento de compra hedônico, enquanto que, nos países menos desenvolvidos, tal tendência seria menos vivenciada pelo consumidor. Sá (2010) reforça a relação existente entre cultura e comportamento de compra, destacando a regionalidade como uma variável que pode influenciar a propensão ao comportamento hedônico ou utilitário do consumidor.

As condutas de consumo hedonista e utilitarista vêm sendo amplamente exploradas em 
pesquisas estrangeiras e nacionais (e.g. FISHBEIN; AJZEN, 1975; HIRSCHMAN; HOLBROOK, 1982; BABIN; DARDEN; GRIFFIN, 1994; HASTREITER; MARCHETTI; PRADO, 1999; ADDIS; HOLBROOK, 2001; LOPES et al, 2010), contudo poucos são os estudos que investigam esses comportamentos de compra nas camadas sociais mais baixas. Peñaloza, Gordiano e Quezado (2011), a exemplo, exploraram o comportamento hedônico e utilitário em consumidores de baixa renda, relacionando esses valores à conduta financeira. A pesquisa de Veloso et al. (2008), por sua vez, aborda o consumo nas classes de baixa renda, investigando as características hedonistas do público infantil.

Brandão, Parente e Oliveira (2010) reafirmam a relevância de estudar os consumidores de baixa renda e sugerem a discussão acerca de um fenômeno de compra comum nos centros de consumo voltados a esse público: o crowding (ou aglomeração em português). Milhares de consumidores de camadas populares vão às compras diariamente, atribuindo uma forte característica de aglomeração às lojas de varejo de baixa renda, o que, segundo Brandão, Parente e Oliveira (2010), pode dificultar o processo de compra, afetando diretamente o comportamento do consumidor.

Inicialmente abordada na Sociologia e na Psicologia (PONS; LAROCHE, 2007), a condição de crowding é capaz de provocar um estado de desconforto psicológico em um indivíduo em um local onde a oferta de espaço não supre sua demanda pessoal (STOKOLS, 1972). Eroglu e Machleit (1990) afirmam que esse fenômeno é resultado do excesso de informação em um ambiente. Na área de marketing, esse conceito passou a ser estudado nas loja para identificar o comportamento do consumidor na condição de crowding (HARRELL; HUTT; ANDERSON, 1980), seja esta provocada por aglomeração de pessoas ou por excesso de produtos em exposição (MACHLEIT; EROGLU, 2000).

Para Pons e Laroche (2007), aspectos culturais influenciam diretamente a percepção do fenômeno de crowding. Consumidores de diferentes países teriam sensações distintas em uma mesma situação de aglomeração em ambiente de loja. Ainda segundo Pons e Laroche (2007), a percepção de crowding também varia dentro de uma mesma cultura, uma vez que a sensação de lotação no varejo pode depender das demais situações de aglomeração vivenciadas por esse consumidor no dia a dia. Nesse sentido, Brandão, Parente e Oliveira (2010) sugerem que os consumidores de menor renda seriam mais tolerantes ao crowding, uma vez que são mais familiarizados com locais pouco espaçosos e com grande aglomeração de pessoas, como os transportes públicos.

Nesse contexto, visando contribuir para o conhecimento da conduta do consumidor de baixa renda, classe de relevante singularidade cultural, este estudo objetiva investigar a percepção de crowding em diferentes centros de varejo popular, relacionando esse fenômeno a dois importantes valores do comportamento de consumo, o hedonismo e o utilitarismo, ainda pouco explorados em consumidores de baixa renda.

Para alcançar esse objetivo, foram escolhidos dois centros comerciais voltados para o consumo das classes populares. O primeiro é um shopping tradicionalmente conhecido em Fortaleza por possuir preços atraentes, localizado em uma região pouco abastada da cidade. $\mathrm{O}$ shopping possui duas praças de alimentação, cinco salas de cinema e aproximadamente 300 lojas, em sua maioria, de marcas populares, recebendo um público médio de 2,5 milhões de consumidores ao mês.

O outro local escolhido foi o Beco da Poeira (Beco), conhecido mercado popular localizado no centro da cidade da Fortaleza, que, nos últimos anos, tem se destacado economicamente, comercializando, principalmente, peças do vestuário e acessórios, constituindo um exemplo de 
varejo que atinge essencialmente as camadas mais pobres da população (MATOS; MOTA, 2006). A principal característica pela qual o Beco é conhecido são seus preços baixos. Segundo Lemos e Junior (2009), essas são suas vantagens competitivas, visto que, na percepção dos clientes, a qualidade é a mesma de lojas convencionais, mas o preço é bem inferior porque os produtos não têm "etiqueta de marca". Para esses autores, o consumidor do Beco busca o preço baixo dos produtos e não a estética do ambiente, que se encontra, por exemplo, nos shopping centers de forma geral.

A escolha dos dois locais justifica-se por serem centros comerciais de baixa renda, igualmente lotados de consumidores e de produtos, mas com estruturas físicas diferentes, o que poderia transmitir, a um mesmo consumidor, diferentes percepções de crowding. Além disso, supõe-se, também, que os dois locais possuem consumidores com comportamentos de compra diferentes, uma vez que o shopping, embora frequentado majoritariamente por pessoas de baixa renda, possui grande variedade de lojas, sendo, assim, um local propenso ao comportamento de compra hedônico. Já no Beco da Poeira, acredita-se que prevaleça o valor de compra utilitário, uma vez que esse centro comercial possui estrutura física precária e é frequentado por consumidores de baixa renda, atraídos pelos baixos preços praticados no local (PEÑALOZA; GORDIANO; QUEZADO, 2011).

A partir do explanado, chegou-se à seguinte questão de pesquisa: como a percepção de crowding é influenciada pelo comportamento hedônico e/ou utilitário do consumidor de baixa renda? Os objetivos deste estudo são, portanto, identificar a relação existente entre crowding e comportamento hedônico e utilitário de compra, bem como verificar a relação entre local de consumo, comportamento hedônico e utilitário e percepção de crowding em consumidores de camadas populares.

\section{REFERENCIAL TEÓRICO}

Esta seção está subdivida em duas partes. No primeiro subitem, são apresentados os principais conceitos sobre valores de consumo hedônico e utilitário, com vistas a sua contextualização aos dois centros de consumo analisados: Beco da Poeira e shopping. No segundo subitem, é discutido o construto de percepção de crowding e suas particularidades.

\subsection{Valores de Compra Hedônico e Utilitário}

Pesquisas que investigam o comportamento do consumidor são de relevante contribuição no campo do marketing (e.g. GRANATO; PEREIRA, 2011; PINTO; LARA, 2008; GONÇALVES FILHO; MELLO; MONTEIRO; MADUREIRA, 2010). O interesse em abordar esses aspectos do consumo se justifica pela necessidade de as organizações conhecerem seus clientes e satisfazê-los, para, consequentemente, permanecerem competitivas no mercado (HASTREITER; MARCHETTI; PRADO, 1999). Estudos sobre comportamento do consumidor, abordando valor de compra, inicialmente, estavam voltados para a visão tradicional de consumo, caracterizado pelo consumo utilitário (D’ANGELO, 2004).

Segundo Addis e Holbrook (2001), esse tipo de consumo é baseado na compra racional e na satisfação de uma necessidade. O valor utilitário de uma compra é reconhecido por fatores como sua utilidade, eficiência e conveniência (ALLEN; NG, 1999), além de ser caracterizado pela procura de menor preço e maior qualidade (ANDERSSON; ENGELBERG, 2006). Com o início dos estudos voltados ao consumo de características emocionais, em que a escolha racional dá lugar a uma visão experiencial, surge uma modalidade denominada de consumo hedônico (HOLBROOK; HIRSCHMAN, 1982).

Definido por características que remetem à sensibilidade, como sons, gostos, impressões táteis e imagens visuais, o consumo hedônico, por sua vez, possui correlação com a experiência do 
processo de compra e não somente com o produto. De acordo com Holbrook e Hirschman (1982), os valores percebidos durante a compra são artefatos simbólicos e decisivos para a motivação da compra. Portanto, diferente do comportamento utilitário, no qual prevalece a compra racional e funcional, o comportamento hedônico é marcado pela presença de aspectos multisensoriais e emocionais e seu principal objetivo é a busca por diversão e prazer (HOLBROOK; HIRSCHMAN, 1982).

O comportamento hedônico e utilitário do consumidor também pode estar relacionado ao local de compra (DONOVAN et al, 1994; COTTET; LICHTLÉ; PLICHON, 2006). O shopping escolhido para esta pesquisa agrega ao ambiente de varejo, além das praças de convivência e cinemas, aspectos hedônicos, de lazer e entretenimento, como exposições culturais e atividades recreativas. Essa diferenciação, através de características multissensoriais do ambiente de varejo, incluindo sons, sensações táteis e aspectos visuais, é reforçada por Addis e Holbrook (2001) como facilitadora do comportamento de compra hedônico por parte do consumidor.

Nos mercados populares, como o Beco escolhido para este estudo, atrativos multissensoriais não são utilizados, uma vez que se trata de locais caracterizados principalmente pela prática de preços baixos (LEMOS; JUNIOR, 2009). Portanto, para o Beco da Poeira e para o shopping, supõe-se que haja propensão ao comportamento utilitário e hedônico, respectivamente, uma vez que, embora detenham cosumidores de perfil econômico similar, são locais diferentes quanto à sua estrutura física.

Nesse contexto, formularam-se as seguintes hipóteses:

H1a: O comportamento de compra dos consumidores do Beco da Poeira é mais utilitário do que hedônico.

H1b: O comportamento de compra dos consumidores do shopping é mais hedônico do que utilitário

H2a: Os consumidores do Beco tendem a ser mais utilitários que os consumidores do shopping.

H2b: Os consumidores do shopping tendem a ser mais hedônicos que os consumidores do Beco.

Tendo em vista a coexistência desses dois valores e o objetivo deste estudo, de identificar a intensidade como que tais valores se apresentam no comportamento de compra dos consumidores do Beco e do Shopping, utilizou-se da escala de valor de compra criada por Babin, Darden e Griffin (1994) e revalidada por Jones, Reynolds e Arnold (2006), que mede o valor utilitário e hedônico. Para esta pesquisa, escolheu-se a escala já adaptada por Lopes et al. (2010), aplicada em duas pesquisas com consumidores brasileiros.

A escala é formada por 13 itens, sete variáveis hedônicas - $(\mathrm{H})$ : H1. Eu me diverti fazendo esta compra; H2. Eu fiz esta compra porque quis e não por obrigação; H3. Comparando com outras coisas que eu poderia ter feito, o tempo gasto comprando este produto foi muito mais agradável; H4. Esqueci os meus problemas enquanto estive neste local; H5. Fazer esta compra foi uma verdadeira distração para mim; H6. Estar neste local foi um jeito muito agradável de passar o tempo; e H7. A busca por este produto me deu um grande prazer - e seis variáveis utilitárias (U): U1. Eu fui eficiente ao fazer esta compra; U2. Eu gastei apenas o tempo necessário para fazer esta compra; U3. Eu fiz esta compra com rapidez; U4. Fazer esta compra foi como cumprir uma obrigação; U5. Foi fácil fazer esta compra porque eu sabia exatamente o que eu queria comprar; e U6. O preço que paguei nesta compra compensou o tempo que gastei. 


\subsection{Crowding}

A partir do desenvolvimento de pesquisas que visam investigar a conduta do consumidor em lojas varejistas (EROGLU; MACHLEIT, 1990; BITNER, 1992, BABIN; DARDEN; GRIFFIN, 1994; MACHLEIT; EROGLU, 2000; GREWAL et al., 2003; PONS; LAROCHE, 2007, BRANDÃO; PARENTE; OLIVEIRA, 2010), observa-se uma forte relação entre o comportamento de compra e os aspectos físicos do ambiente de varejo. Haja vista a crescente concentração de clientes nas lojas do setor varejista, destaca-se, nessas pesquisas, a discussão em torno do fenômeno de crowding, classificado por Stokols (1972) como um desconforto psicológico sentido por um indivíduo em um local abarrotado.

Sobre a percepção desse fenômeno em lojas de varejo, Machleit e Eroglu (2000) afirmam que a sensação de crowding ocorre quando, em um espaço limitado, o volume de pessoas, objetos ou ambos restringe a atividade do consumidor ou a realização do objetivo de compra. Essas duas condições de crowding, causadas pela densidade de pessoas ou objetos, compõem um conceito multidimensional sob o ponto de vista do consumidor em um ambiente de loja. A condição de crowding, a partir da densidade humana, é reflexo da dificuldade, ou até do impedimento, da escolha de compra devido à aglomeração formada por outros consumidores na loja. A condição de densidade espacial, por sua vez, ocorre quando esse impedimento é consequência de um volume excessivo de produtos em exposição, impossibilitando o consumidor de encontrar um produto ou de se locomover na loja (HUI; BATESON, 1991).

Segundo Rompay et al. (2008), a partir de resultados de uma pesquisa norte-americana, pode-se verificar que a sensação de crowding dos consumidores mostrou-se mais intensa quando houve densidade espacial do que em casos de densidade humana. Contudo, no Brasil, a densidade humana parece incomodar mais os consumidores do que a espacial. Tal fato pode ser resultado da lentidão no atendimento, característica moderadora da percepção de crowding e muito comum em lojas de varejo brasileiras (BRANDÃO; PARENTE; OLIVEIRA, 2010), especialmente naquelas voltadas para o consumidor de baixa renda.

Dessa forma, a percepção de aglomeração tem relação com a cultura de cada país e com a experiência de cada indivíduo. Dois consumidores em um mesmo ambiente de varejo podem ter diferentes pontos de vista em relação ao nível de crowding (MACHLEIT; EROGLU, 2000). A avaliação de densidade para cada consumidor depende de suas concepções próprias e de sua disposição a interagir com o meio em que se encontra. Ocorre o fenômeno de crowding quando o resultado dessa avaliação é negativo e a densidade percebida é disfuncional, confinadora e restritiva (EROGLU; MACHLEIT, 1990).

A percepção de crowding também pode depender da motivação do consumidor à compra: por tarefa orientada (consumo utilitário) e por tarefa não orientada (consumo hedônico) (TELLER, 2008). O consumidor utilitário tem um objetivo de compra predeterminado a ser cumprido em um curto período de tempo. Dessa forma, em um ambiente de varejo lotado, esse tipo de consumidor teria uma maior sensação de crowding devido ao maior dispêndio de tempo para a realização da compra (EROGLU; HARRELL, 1986).

O consumidor com perfil utilitarista, que também é caracterizado por visar à poupança (BARDHI; ARNOULD, 2005), pode sentir-se atraído a entrar em uma loja de varejo lotada e desordenada, uma vez que essas características transmitem uma imagem de local de baixo preço (LANGREHR, 1991). No entanto, de acordo com esse autor, esse aspecto é irrelevante, uma vez que, dentro da loja, o consumidor utilitário tende a se sentir nervoso, tenso, confuso e pouco confiante sobre a realização da compra.

Já o consumidor hedônico, em um ambiente de loja com um número excessivo de gôn- 
dolas e prateleiras abarrotadas, sente-se estimulado à compra, uma vez que é mais propenso à compra não planejada (BABIN; DARDEN; GRIFFIN, 1994). Sob outro aspecto, o consumidor de caráter hedonista, por não sofrer com a pressão do tempo, não parece se sentir incomodado em uma loja lotada com outros clientes (EROGLU; HARRELL, 1986). Assim, características da atmosfera de varejo que levariam à percepção de crowding por utilitaristas podem chegar a ser positivamente incitantes para os hedonistas (EROGLU; MACHLEIT, 1990).

Diante do exposto, foram formuladas as seguintes hipóteses:

H3a: Há uma correlação negativa entre comportamento de compra hedônico e percepção de crowding humano e espacial.

H3b: Há uma correlação positiva entre comportamento de compra utilitário e percepção de crowding humano e espacial.

H4: Os consumidores do shopping tendem a ter uma menor percepção do crowding (humano e espacial) do que os consumidores do Beco da Poeira.

H5: Os consumidores (do shopping e do Beco) percebem mais o crowding humano do que o crowding espacial.

Para testar essas hipóteses, utilizou-se da escala original desenvolvida por Machleit, Kellaris e Eroglu (1994) de Percepção de Crowding Humano e Espacial (a tradução dos itens da escala original para a língua portuguesa foi realizada por dois tradutores independentes).

A escala é formada por oito itens, quatro variáveis que medem a sensação de crowding humano - $(\mathrm{CH})$ : $\mathrm{CH} 1$. O local em minha opinião está muito lotado; $\mathrm{CH} 2$. O local está um pouco ocupado demais; $\mathrm{CH} 3$. Houve muito tráfego de clientes no local durante a minha visita; $\mathrm{CH} 4$. Há muitos clientes no local - e quatro variáveis que medem a percepção de crowding espacial - (CE): CE1. O local parece muito espaçoso; CE2. Eu tenho a sensação de aperto/pouco espaço comprando neste local; CE3. O local passa a sensação de amplitude; CE4. O local faz com que os clientes se sintam confinados.

\section{METODOLOGIA DE PESQUISA}

Este estudo possui natureza quantitativa e caráter descritivo, pois busca descrever uma determinada população a partir das relações entre as suas variáveis. Por ser uma pesquisa realizada em centros de consumo frequentados por consumidores de baixa renda, um cuidado adicional é necessário, visto que as metodologias tradicionais são geralmente desenvolvidas para consumidores com alto grau de instrução (ROCHA; SILVA, 2009).

Tendo em vista essa limitação, as entrevistas foram realizadas por pesquisadores que conhecem e frequentam esses lugares: no Beco da Poeira aos sábados, das $8 \mathrm{~h}$ às $14 \mathrm{~h} 30 \mathrm{~min}$; e no shopping em finais de semana e horários de maior afluência de público, das $14 \mathrm{~h}$ às $19 \mathrm{~h}$. 0 tempo de preenchimento dos questionários foi de, aproximadamente, 10 a 12 minutos. Foram coletados em quatro dias, totalizando 404 questionários aplicados.

Foi utilizada uma amostra de natureza não probabilística por conveniência. Uma amostra probabilística que fosse representativa do Beco e do shopping não seria possível, uma vez que não existe nenhuma informação documentada sobre número e perfil de consumidores desses lugares.

O instrumento de pesquisa foi composto por cinco partes. As escalas aplicadas foram homogeneizadas e todas as perguntas realizadas em escala de Lickert de 0 a 10 pontos (com 0 
representando total discordância e 10 total concordância). Presumiu-se que as pessoas estariam mais familiarizadas com essa escala, o que diminuiria o erro nas respostas.

O questionário aplicado foi composto de 26 enunciados, no qual as 21 primeiras questões correspondiam às duas escalas utilizadas. A primeira escala, composta por treze enunciados, buscava caracterizar o comportamento de compra do consumidor em hedônico ou utilitário. A segunda escala utilizada, composta por oito enunciados, media a percepção de crowding humano e espacial. Os outros cinco enunciados buscavam caracterizar os respondentes segundo sexo, idade, escolaridade, bairro de residência e níveis de renda.

Vale destacar que pré-testes foram aplicados especificamente com consumidores de baixa escolaridade do Beco e do shopping para assegurar o entendimento das perguntas e das escalas. Essa forma de abordagem fez com que houvesse pouquíssimos dados não respondidos, que foram tratados utilizando o método de substituição pela média.

Os dados foram analisados com o auxílio do Software Statistical Package for the Social Sciences (SPSS 18.0), de módulos de estatística descritiva, da análise fatorial, da correlação de $P e-$ arson e da Análise de Variância (ANOVA). Por meio da análise exploratória dos dados, observa-se que quase não houve missing, como foi assinalado anteriormente. Os outliers foram verificados para o caso da renda e idade, sendo excluídos dos questionários.

Por meio da estatística descritiva, a população estudada foi caracterizada. Posteriormente, para definir os construtos de valores de consumo e crowding, foi realizada a análise fatorial exploratória. Em seguida, foram obtidas as médias de cada um dos fatores resultantes para cada local (shopping e Beco) e confirmadas via ANOVA. Por fim, uma análise das correlações entre os fatores foi realizada por meio da técnica de correlação de Pearson.

\section{ANÁLISE DOS RESULTADOS}

Nesta seção, é apresentada a análise dos resultados encontrados nos dois estudos empíricos a partir dos tratamentos estatísticos utilizados.

\subsection{Análise Descritiva}

Como esperado, a amostra do Beco foi constituída por $70,5 \%$ de mulheres e $29,5 \%$ de homens, com idades variando entre 15 e 73 anos (média de 33 anos). Os frequentadores do Beco vêm de bairros mais afastados, alguns de outras cidades ou outros estados. Com relação ao grau de instrução, mais da metade dos entrevistados (57\%) declararam ter o ensino médio completo, 13 pessoas $(6,5 \%)$ declararam ter nível superior completo e 73 pessoas afirmaram $(36,5 \%)$ não terem concluído o ensino fundamental ou médio.

No shopping, assim como no Beco, as mulheres foram maioria (60,3\% mulheres e $39,7 \%$ homens). A faixa etária dos entrevistados variou entre 14 e 81 anos, sendo a média de 31 anos. Os frequentadores do shopping também vêm de bairros mais afastados da cidade e poucos moram nos bairros mais abastados. Quanto ao grau de instrução, este foi maior que o esperado: 117 entrevistados $(57,3 \%)$ declararam ter o ensino médio completo, 41 pessoas $(20,1 \%)$ declararam ter nível superior completo e apenas 46 pessoas (22,5\%) disseram não ter terminado o ensino fundamental ou médio.

No que tange à renda familiar dos entrevistados do Beco, $50 \%$ pertence a famílias que ganham até dois salários mínimos, o quartil inferior ganha até 1,5 salário mínimo e o quartil superior ganha mais de três salários mínimos, sendo a média salarial das famílias dos entrevistados de 2,9 salários mínimos mensais. No shopping, 50\% das pessoas pertencem a famílias que ganham 
até três salários mínimos, o quartil inferior ganha até dois salários mínimos e o quartil superior ganha mais de quatro salários mínimos, sendo a média salarial de 3,9 salários mínimos mensais.

A partir da classificação do IBGE (2010), 48\% dos respondentes desta pesquisa pertencem à classe $E, 34 \%$ pertencem à classe $D, 16 \%$ à classe $C$ e somente $2 \%$ às classes $A$ e $B$. Assim, caracteriza-se a amostra desta pesquisa como majoritariamente de baixa renda. Essa caracterização, ratificada por Prahalad (2005), é também utilizada por Kotler e Lee (2010) para segmentação do mercado de baixa renda brasileiro e pelo setor empresarial especializado na baixa renda.

\subsection{Análise Fatorial, Análise de Variância Univariada (ANOVA) e Correlação de Pearson}

Inicialmente, foi realizada uma análise fatorial com a escala de comportamento de consumo hedônico e utilitário com os dados do shopping e do Beco. A escala mostrou-se apropriada quando eliminados os itens $\mathrm{H} 1$. Eu me diverti fazendo esta compra, H2. Eu fiz esta compra porque quis, e não por obrigação, U4. Fazer esta compra foi como cumprir uma obrigação, U5. Foi fácil fazer esta compra porque eu sabia exatamente o que eu queria comprar e U6. O preço que paguei nesta compra compensou o tempo que gastei, uma vez que apresentaram comunalidades inferiores a 0,5. Esses resultados são condizentes com outros estudos (LOPES et al., 2010; PEÑALOZA; GORDIANO; QUEZADO, 2011).

O teste de esfericidade de Bartlett foi significativo ao nível de 0,1\% ( $\chi 2=1580,665 ; 28$ $\mathrm{gl} ; \mathrm{p}<0,01$ e o teste de Kaiser-Meyer-Olkin (KMO), mostrando-se superior a 0,8 (KMO=0,817). Usando a Rotação Varimax e o critério de autovalores superiores a um, conforme recomendam Hair et al. (2005), foram extraídos dois fatores que, em conjunto, explicaram $69,1 \%$ da variância total e indicaram uma boa confiabilidade, atestada pelo Alpha de Cronbach de $\alpha=0,865$ para o primeiro fator, que representa o valor de compra hedônico, e $\alpha=0,801$ para o segundo fator, que representa o valor de compra utilitário (TAB. 1).

TABELA 1 - Matriz de Componentes Rotacionada Comportamento de Consumo

\begin{tabular}{lcc}
\hline & \multicolumn{2}{c}{ Fatores } \\
Itens & Hedônico & Utilitário \\
\hline H5. Fazer esta compra foi uma verdadeira distração para mim. & 0,89 & 0,85 \\
H4. Esqueci os meus problemas enquanto estive nesse local. & 0,82 \\
H6. Estar nesse local foi um jeito muito agradável de passar o tempo. & 0,70 & 0,87 \\
H7. A busca por este produto me deu um grande prazer. & 0,68 & 0,86 \\
H3. Comparando com outras coisas que eu poderia ter feito, o tempo & 0,74 \\
gasto comprando este produto foi muito mais agradável. & 29,1 \\
U3. Eu fiz esta compra com rapidez. & \\
U2. Eu gastei apenas o tempo necessário para fazer esta compra. & 0,80 \\
U1. Eu fui eficiente ao fazer esta compra. & 0,86 \\
\hline Variância Explicada (\%) & \\
Alpha de Cronbach & \\
\hline Fonte: SPSS & \\
Extraction Method: Principal Component Analysis. Rotation Method: Varimax with Kaiser \\
Normalization.
\end{tabular}

Outra análise fatorial foi realizada com a escala que mede a percepção de crowding em relação às dimensões humana e espacial. A escala mostrou-se apropriada após a exclusão do item $\mathrm{CH} 2$. Esse local está um pouco ocupado demais, pela mesma razão já explanada (comunali- 
dade inferior a 0,5).

Conforme proposto pelos autores que desenvolveram a escala de crowding (MACHLEIT; KELLARIS; EROGLU, 1994), os itens CE1. Esse local parece muito espaçoso e CE3. Esse local passa a sensação de amplitude tiveram seus números invertidos para que todos os itens que compõem a escala tivessem uma direção homogênea. Após, os itens ficaram agrupados em dois fatores, denominados "crowding humano" e "crowding espacial" (TAB. 2).

$O$ teste de esfericidade de Bartlett assumiu valores de 931,74 , significativo a 0,01\%, e o teste de KMO resultou em um coeficiente de 0,667. A escala foi composta por dois fatores, que, em conjunto, explicam $64,3 \%$ da variância total. A confiabilidade da escala, medida pelo Alpha de Cronbach, apresentou valores satisfatórios $(0,77$ e 0,72, para o fator crowding humano e crowding espacial, respectivamente).

TABELA 2 - Matriz de Componentes Rotacionada Crowding

\begin{tabular}{lcc}
\hline Itens & $\begin{array}{c}\text { Fatores } \\
\text { Crowding } \\
\text { Espacial }\end{array}$ \\
\hline CH3. Houve muita circulação de clientes por esse local durante a minha visita. & 0,82 \\
Humano & 0,80 \\
CH4. Há muitos clientes nesse local. & 0,78 \\
CH1. Esse local em minha opinião está muito lotado. & 0,82 \\
CE1. Esse local parece muito espaçoso. (R) & 0,77 \\
CE3. Esse local passa a sensação de amplitude. (R) & 0,65 \\
CE2. Eu tenho a sensação de aperto/pouco espaço comprando nesse local. & 0,64 \\
CE4. Esse local faz com que os clientes se sintam confinados. & 30,4 \\
\hline Variância Explicada (\%) & 33,9 \\
Alpha de Cronbach & 0,77 & 0,72 \\
\hline
\end{tabular}
Fonte: SPSS

Extraction Method: Principal Component Analysis. Rotation Method: Varimax with Kaiser Normalization.

a. Rotation converged in 3 iterations.

Com base nos resultados da análise fatorial para as escalas de comportamento de compra e percepção de crowding, foram calculadas as médias de cada um dos quatro fatores para os dois locais em que a pesquisa foi realizada: shopping e Beco da Poeira (TAB. 3). Prosseguiu-se com a Análise de Variância Univariada (ANOVA) para verificar se as diferenças entre as médias dos dois locais são significativas. Por meio dessa técnica, verificou-se que os resultados de três, dos quatros fatores, diferem dependendo do local onde a escala foi aplicada.

Os resultados da ANOVA mostraram que as diferenças entre as médias nos dois locais são significativas para os fatores de comportamento hedônico, percepção de crowding humano e espacial $(p<0,01)$. O mesmo resultado não foi encontrado para o fator que explicava o comportamento de consumo utilitário, o que já era esperado, pois apresentaram médias parecidas, podendo indicar que os dois locais são igualmente utilitários.

TABELA 3 - Médias dos fatores e ANOVA

\begin{tabular}{lcccc}
\hline Fatores & \multicolumn{2}{c}{ Médias } & \multicolumn{2}{c}{ ANOVA } \\
& Shopping & Beco & F & Sig. \\
\hline Hedônico & 7,72 & 6,87 & 15,36 & 0,00 \\
Utilitário & 7,75 & 7,46 & 1,80 & 0,18
\end{tabular}




\begin{tabular}{llccc} 
Hedônico-Utillitário & $\mathbf{- 0 , 0 3}$ & $\mathbf{- 0 , 5 9}$ & $\mathbf{5 , 2 2}$ & $\mathbf{0 , 0 2}$ \\
Crowding Humano & 8,32 & 8,78 & 8,17 & 0,00 \\
Crowding Espacial & 3,90 & 5,68 & 75,86 & 0,00 \\
Crowding Humano-Crowding Espacial & $\mathbf{4 , 4 1}$ & $\mathbf{3 , 1 0}$ & $\mathbf{2 9 , 4 4}$ & $\mathbf{0 , 0 0}$ \\
\hline
\end{tabular}

Fonte: SPSS

Observando as médias resultantes dos fatores da análise fatorial para o Beco da Poeira, percebe-se um comportamento de compra mais utilitário do que hedônico por parte dos entrevistados (média de -0,59). Isso pode ser explicado devido ao fato de o Beco da Poeira ser um local com preços baixos, o que atrai os consumidores com perfil utilitarista. Dessa forma, encontraram-se evidências que suportam a hipótese $\mathrm{H}_{1 \mathrm{a}}$.

Com relação ao shopping, percebemos que os entrevistados também se mostraram mais propensos ao comportamento de compra utilitário (média de -0,03), rejeitando-se, portanto, a hipótese $\mathrm{H}_{1 \mathrm{~b}}$. Embora o shopping seja um local que induziria as pessoas a um comportamento de compra predominantemente hedônico, os resultados indicaram que os consumidores de baixa renda não abandonaram a característica fortemente utilitária de procurar preços baixos.

Comparando-se os dois locais com relação ao comportamento de compra dos seus clientes, percebe-se que os consumidores do shopping são mais hedônicos que os do Beco, corroborando, portanto, a hipótese $\mathrm{H}_{2 \mathrm{a}}$. As atividades diferenciadas de entretenimento e lazer que o shopping oferece podem ter influenciado o resultado, pois, segundo Holbrook e Addis (2001), essas características, ausentes no Beco, são facilitadores do comportamento de compra hedônico por parte do consumidor.

Em relação ao comportamento de compra utilitário, a diferença entre a média dos dois locais não se mostrou significativa, indicando que os consumidores do Beco e do shopping são similares quanto a características utilitárias. Dessa forma, não foram encontradas evidências que corroborassem a hipótese $\mathrm{H}_{2 \mathrm{~b}}$.

Utilizou-se, ainda, a correlação de Pearson para medir o grau da correlação (e a direção dessa correlação, se positiva ou negativa) entre os fatores (TAB.4).

TABELA 4 - Correlação de Pearson

\section{Coeficientes de Correlação}

\begin{tabular}{ccccc} 
Fatores & Hedônico & Utilitário & Crowding Humano & Crowding Espacial \\
\hline Hedônico & 1 & & & \\
Utilitário & $0,38^{* *} 0,00$ & 1 & 1 & \\
Crowding Humano & $0,12^{*} 0,02$ & $0,09 \quad 0,09$ & $0,19 * * 0,00$ & 1 \\
Crowding Espacial & $-0,36^{* *} 0,00$ & $-0,24^{* *} 0,00$ & 0,00 & \\
\hline
\end{tabular}

Fonte: SPSS

** Correlation is significant at the 0.01 level (2-tailed).

* Correlation is significant at the 0.05 level (2-tailed).

Analisando as correlações entre comportamento de compra e percepção de crowding humano e espacial, observou-se que há uma correlação negativa entre comportamento hedônico e percepção de crowding espacial, bem como uma correlação positiva entre comportamento hedônico e percepção de crowding humano. Assim, foram encontradas evidências parciais que comprovam a hipótese $\mathrm{H}_{3 \mathrm{a}^{\prime}}$ pois era esperado que ambas as correlações fossem negativas. 
Já a hipótese $\mathrm{H}_{3 b}$ não pôde ser totalmente testada, pois a correlação entre comportamento de compra utilitário e crowding humano não se mostrou significativa $(p>0,05)$. No que se refere ao consumo utilitário e crowding espacial, a hipótese mostrou-se inválida, pois entre esses dois fatores existe uma correlação negativa e não positiva, como era esperado. No entanto, observa-se que a correlação negativa entre crowding espacial e comportamento de consumo apresentou-se maior no fator hedônico do que n o fator utilitário.

Verificou-se, ainda, que os consumidores do shopping perceberam menos o crowding humano e espacial do que os consumidores do Beco, conforme esperado. Desse modo, encontraram-se evidências que validam a hipótese $\mathrm{H}_{4}$. Esse resultado pode ser explicado pela infraestrutura mais confortável do shopping e pelo perfil menos hedônico dos consumidores do Beco.

Vale ressaltar que, em ambos os locais, shopping e Beco, os consumidores percebem com mais intensidade o crowding humano do que o crowding espacial. Esses resultados corroboram a hipótese $\mathrm{H}_{5}$, de que a percepção de crowding humano é mais intensa do que a percepção de crowding espacial. Brandão, Parente e Oliveira (2010) encontraram as mesmas evidências: no Brasil os consumidores tendem a se incomodar mais com a aglomeração de pessoas do que com a aglomeração decorrente de uma infraestrutura inadequada.

TABELA 5 - Resultados das Hipóteses

\begin{tabular}{ll}
\hline Hipóteses & Resultados
\end{tabular}

H1a O comportamento de compra dos consumidores do Beco da Poeira é mais utilitário do que hedônico.

H1b O comportamento de compra dos consumidores do shopping é mais hedônico do que utilitário.

H2a Os consumidores do Beco tendem a ser mais utilitários que os consumidores do shopping.

H2b Os consumidores do shopping tendem a ser mais hedônicos que os consumidores do Beco.

H3a Há uma correlação negativa entre comportamento de compra hedônico e percepção de crowding humano e espacial.

H3b Há uma correlação positiva entre comportamento de compra utilitário e percepção de crowding humano e espacial.

H4 Os consumidores do shopping tendem a ter uma menor percepção do crowding (humano e espacial) do que os consumidores do Beco da Poeira.

H5 Os consumidores (shopping e Beco) percebem mais o crowding humano em relação ao crowding espacial.

Fonte: Elaborada pelos autores.

\section{CONSIDERAÇÕES FINAIS}

Este estudo buscou explorar padrões culturais de consumo nas camadas sociais mais baixas, relacionando o fenômeno de crowding, comum no varejo de baixa renda, aos valores de compra hedônico e utilitário dos consumidores que frequentam os dois locais escolhidos para a pesquisa empírica.

Ao contrário do esperado, os indivíduos participantes da amostra do shopping e do Beco da Poeira mostraram-se igualmente propensos ao comportamento utilitário. No entanto, os clientes do shopping mostraram-se mais hedônicos do que os consumidores do Beco, em

\section{Aceita}

Rejeitada

Aceita

Rejeitada

Parcialmente Aceita/Parcialmente Rejeitada

Parcialmente não pôde ser comprovada/ Parcialmente Rejeitada

Aceita

Aceita 
concordância com os estudos de Donovan et al. (1994) e Cottet, Lichtlé e Plichon (2006), que caracterizam os shoppings como locais de estrutura física propensa ao comportamento de compra hedônico.

Quando as variáveis de comportamento utilitário e hedônico foram unidas em um único fator, percebeu-se que os consumidores do shopping são levemente propensos ao utilitarismo. Isso pode ser justificado pelo fato de o shopping escolhido para esta pesquisa ser frequentado essencialmente por consumidores de baixa renda, que procuram por menores preços, forte característica da compra utilitária (BARDHI; ARNOULD, 2005).

No Beco, quando as variáveis hedônicas e utilitárias foram unidas para gerar um único valor, percebeu-se claramente que os consumidores possuem um forte perfil utilitarista. No artigo de Babin, Darden e Griffin (1994) sobre a relação existente entre cultura e valor de compra, sugere-se que os consumidores de países menos desenvolvidos são mais propensos ao consumo utilitário, o que pode ser explicado, dentre outros aspectos, pelo fato de grande parte da população desses países ser constituída por camadas de baixa renda.

Os resultados desta pesquisa revelaram evidências de que, apesar de as pessoas de baixa renda serem caracterizadas pelo perfil utilitarista de consumo, um local com infraestrutura mais hedônica parece ter influência positiva no comportamento do consumidor, que sente mais prazer na realização da compra em um ambiente de loja atraente.

Além do local de consumo, este estudo também relaciona o valor de compra à percepção de crowding. Na maioria dos estudos sobre crowding, os consumidores mostraram-se menos satisfeitos com a experiência de compra à medida que o incremento da densidade humana e espacial era maior (EROGLU; HARELL, 1986; HUI; BATESON, 1991; MACHLEIT; EROGLU, 2000; ROMPAY et al., 2008). Entretanto, para Baker et al. (1994), diante da situação de crowding caudado pela dimensão humana, o consumidor infere algumas carcaterísticas positivas em relação ao local de compra, como, por exemplo, preço e qualidade do produto.

No Brasil, locais como a Rua 25 de março, em São Paulo, a Feira dos Importados, em Brasília, e o próprio Beco da Poeira, em Fortaleza, são famosos pela aglomeração humana e espacial e, ainda assim, são exemplos bem-sucedidos de comércio popular. Pons e Laroche (2007) encontraram em seu estudo uma correlação positiva entre satisfação de compra e crowding, atribuindo esse resultado a variáveis como cultura e busca de produtos supérfluos (valor hedônico de compra).

Apesar de este estudo limitar-se a comparar valor de compra com percepção de crowding, não abordando a satisfação do consumidor, sugestão para investigações futuras, seu diferencial está no fato de que estuda esses dois fenômenos e sua relação em setores de baixa renda em dois locais de compra com diferentes infraestruturas. Além disso, difere-se por ter sido aplicado na Região Nordeste do país e, mais especificamente, em um mercado de consumo popular de grande destaque econômico e cultural na região.

Foram encontradas evidências de que consumidores do Beco da Poeira, cuja estrutura física é deficiente, perceberam mais crowding (humano e espacial) do que os consumidores do shopping, onde há uma infraestrutura adequada para atender a quantidade de pessoas que frequentam o local, mesmo nos dias de maior fluxo de clientes.

No que se refere ao valor de compra hedônico e/ou utilitário e à percepção de crowding, de uma forma geral, as pessoas sentiram-se incomodadas com o crowding humano e espacial, independente do comportamento de consumo. No entanto, quanto maiores eram as características hedônicas do consumidor, menor era a sua percepção de crowding espacial.

Outro resultado encontrado sugere que os entrevistados, tanto do Beco quanto do sho- 
pping, percebem bem mais o crowding causado pela densidade humana do que o causado pela densidade espacial. Outros autores (BRANDÃO; PARENTE; OLIVEIRA, 2010) encontraram resultados similares, evidenciando que, no Brasil, a percepção de crowding na densidade humana é maior do que na densidade espacial, em contraposição a estudos realizados no mercado norte-americano (MACHLEIT, 2000), em que o desconforto espacial é maior que o desconforto humano.

A análise dos dados desta pesquisa revelou que existem algumas carcaterísticas no fenômeno de crowding que parecem variar de cultura para cultura. Assim, este trabalho contribui para enriquecer os arcabouços teóricos ligados ao fenômeno de crowding e ajuda a estabelecer novas práticas que auxiliam o empresário varejista, principalmente os voltados para a baixa renda, a lidar com essa situação.

Por fim, propõe-se que, em outros estudos, sejam feitas comparações entre locais de baixa e alta renda e a percepção de crowding, pois alguns autores (BRANDÃO; PARENTE; OLIVEI$R A, 2010)$ sugerem que os consumidores de alta renda possuem uma menor tolerância a aglomerações e, ao realizarem suas compras, fazem-no com a ideia de obter bens exclusivos em locais que dificilmente estarão sujeitos ao fenômeno de crowding.

\section{REFERÊNCIAS}

ADDIS, M.; HOLBROOK, M. B. On the conceptual link between mass customization and experiential consumption: an explosion of subjectivity. Journal of Consumer Behavior, v.1, n.1, p. 50-67, 2001.

ALLEN, M. W.; NG, S. H. The Direct and Indirect Influences of Human Values on Product Ownership. Journal of Economic Psychology, v. 20, p. 5-39, 1999.

ANDERSSON, P.; ENGELBERG, E. Affective and rational consumer choice modes: The role of intuition, analytical decision-making, and attitudes to money. Working Paper in Business Administration, n.13, 2006.

ARAUJO, M. C. B. de. História de Beco: Quando a poeira assenta entrevemos rosto, punho e corações. Fortaleza, CE: [s.n.], 2010. Disponível em :<http://issuu.com/mayara.carol /docs/ historiasdebeco>. Acesso em: 10 dez. 2011.

BABIN, B. J.; DARDEN, W. R.; GRIFFIN, M. Work and/or Fun: Measuring Hedonic and Utilitarian Shopping Value. Journal of Consumer Research, v. 20, n. 4, p. 644-656, 1994.

BARDHI, F; ARNOULD, E. J. Thrift shopping: Combining utilitarian thrift and hedonic treat benefits. Journal of Consumer Behavior, v. 4, n. 4, p. 223-233, 2005.
BITNER, M. J. Service's capes: the impact of physical surroundings on customers and employees. Journal of Marketing, v. 56, p. 57-71, Apr. 1992.

BRANDÃO, M. M.; PARENTE, J.; OLIVEIRA, B. B. Percepção de crowding no varejo: uma investigação exploratória no mercado brasileiro. Revista Eletrônica em Administração-RAE, v. 9, n.. 2, 2010.

COTTET, P.; LICHTLÉ, M. C.; PLICHON, V. The role of value in services: a study in a retail environment. Journal of Consumer Marketing, v. 23, n. 4, p. 219-227, 2006.

D'ANGELO, A. C. Valores e significados do consumo de produtos de luxo. 2004. Dissertação (Mestrado Acadêmico em Administração) - Universidade Federal do Rio Grande do Sul. Programa de Pós-Graduação em Administração. Escola de Administração, Porto Alegre, 2004. Disponível em:< http://hdl.handle. net/10183/4845>. Acesso em: 02 nov. 2011.

DONOVAN, R.J.; ROSSITER, J.R.; MARCOOLYN, G.; NESDALE, A. Store atmosphere and purchasing behavior. Journal of Retailing, v. 70, 283-294. 1994.

EROGLU, S. A.; HARRELL, G.D. Retail Crowding: theoretical and strategic implications. Journal of Retailing, v. 62, n. 4, p. 349-362, 1986.

EROGLU, S. A.; MACHLEIT, K. A. An Empirical Study of Retail Crowding: Antecedents and Consequences. 
Journal of Retailing, v. 66, n. 2, 1990.

FISHBEIN, M.; AJZEN, I. Belief, attitude, intention and behavior: an introduction to theory and research. Reading. Massachusetts: Addison - Wesley, 1975.

GONÇALVES FILHO, C.; MELLO, E. F.; MONTEIRO, P.R.; MADUREIRA, K.T. Lealdade intencional e comportamento: influências contextuais e individuais no setor de telefonia móvel póspaga. Revista de Administração da UFSM, v. 3, n. 3, p. 441-458, 2010.

GRANATO, L.A.C.; PEREIRA, P.F.P. Ainda existem consumidores fiéis? Revista de Administração da UFSM, v. 4, n. 1, p. 9-18, 2011.

GREWAL, D.; BAKER, J.; LEVY, M.; VOSS, B.G. The effects of wait expectations and store atmosphere evaluations on patronage intentions in service-intensive retail stores. Journal of Retailing. v. 79, p. 259-268, 2003.

HASTREITER, S.; MARCHETTI, R.; PRADO, P. Tipologia de Consumidores baseada nas Razões e Motivações de Freqüência em Shopping Centers. In: do XXIII Encontro Nacional de programas de pós-graduação. Anais... Rio de Janeiro, RJ, 1999.

HARRELL, G.D.; HUTT, M.D.; ANDERSON, J.C. 'Path analysis of buyer behavior under conditions of crowding, Journal of Marketing Research, n. 17, p. 45-51, 1980.

HERNANDEZ, J. M. C. Foi Bom para Você? Uma Comparação do Valor Hedônico de Compras Feitas em Diferentes Tipos de Varejistas. RAM - Revista de Administração Mackenzie, v. 10, n. 2, p. 11-30, 2009.

HOLBROOK, M. B.; HIRSCHMAN, E. C. The Experiential Aspects of Consumption: Consumer Fantasies, Feelings, and Fun. Journal of Consumer Research, v. 9, n. 2, p. 132-140, 1982.

HUI, M. K.; BATESON, J. E. G.. Perceived Control and the Effects of Crowding and Consumer
Choice on the Service Experience. Journal of Consumer Research, v. I, n. 1, p. 174-184, 1991.

JONES, M. A.; REYNOLDS, K. E.; ARNOLD, M. $J$. Hedonic and utilitarian shopping value: investigating differential aspects on retail outcomes. Journal of Business Research, v. 59, n. 9, p. 974-981, 2006.

KOTLER, P.; LEE, N. R. Marketing contra a pobreza: as ferramentas da mudança social para formuladores de políticas, empreendedores, ONGs, empresas e governos. Porto Alegre: Bookman, 2010.

LANGREHR, F. W. Retail shopping mall semiotics and hedonic consumption. Advances in Consumer Research, v. 18, p. 428-433, 1991.

LEMOS, A. Q.; JÚNIOR, E. P. L. Estudo das estratégias de microempreendedores para a obtenção de vantagens competitivas em um complexo organizacional. Revista Brasileira de Gestão e Desenvolvimento Regional, v. 5, n. 2, p. 34-63, 2009.

LOPES, E. L. ; TEIXEIRA, J. M. ; HERNANDEZ, J. M. C. ; MORETTI, S. L. A. . Valores De Compra Hedônico e Utilitário: Duas Aplicações No Varejo Especializado. In: XXXIV ENCONTRO ANUAL DA ANPAD, 2010, Rio de Janeiro. Anais... Rio de Janeiro, v. 1, p. 803, 2010.

MACHLEIT, K.A.; KELLARIS, J.J.; EROGLU, S.A. Human versus spatial dimensions of crowding perceptions in retail environments: a note on their measurement and effect on shopper satisfaction. Mark Lett, p. 183-94, 1994.

MACHLEIT, K.A.; EROGLU, S.A. Perceived retail crowding and shopping satisfaction: what modifies the relationship. Journal Consum Psychol, p. 29-42, 2000.

MATOS, A. L. B.; MOTA, M. D. B. Caminhando pelas ruas do beco, para democrático encontro com a moda. In: I ENCUENTRO LATINOAMERICANO DE DISEÑO “DISEÑO EN PALERMO" COMUNICACIONES ACADÉMICAS, 2006, Buenos Aires. Actas de diseño. Buenos 
Aires, v.1, p.171-172. Disponível em:< http://fido.palermo.edu/servicios_dyc/ publicacionesdc/archivos/13_libro.pdf $>$. Acesso em: 02 nov.2011.

PEÑALOZA, V.; GORDIANO, E.; QUEZADO, I. Consumo Utilitario y Consumo Hedónico en el Beco da Poeira. In: Asamblea Anual de CLADEA (Consejo Latinoamericano de Escuela de Administración), 46., 2011, San Juan Anais... San Juan, Porto Rico, 2011.

PINTO, M.R.; LARA, J.E. O que se publica sobre comportamento do consumidor no Brasil, afinal? Revista de Administração da UFSM, v. 1, n. 3, p. 85-100, 2008.

PONS, F.; LAROCHE, M. Cross-cultural differences in crowd assessment. Journal of Business Research, v.60, p. 269-276, 2007.

PRAHALAD, C. K. A Riqueza na Base da Pirâmide: Como Erradicar a Pobreza com o Lucro. Porto Alegre: Bookman, 2005.

ROCHA, A.; SILVA, J. C. F. da. Consumo na Base da Pirâmide: Estudos Brasileiros. Rio de Janeiro: Mauad X, 2009.

ROMPAY, T.; VAN, J.L.; GALETZKA, M.; PRUYN, A.; GARCIA, J.M.; Human and spatial dimensions of retail density: revisiting the role of perceived control. Psychol Mark, p. 319-35, 2008.

SÁ, A. F. de. Consumo na Sociedade Contemporânea: uma Reflexão Sobre os Motivos que Levam ao Consumo Hedônico In: XXXI Congresso Brasileiro de Ciências da Comunicação, 2010, Caxias do Sul. Anais... Caxias do Sul, RS, 2010.

SARAIVA, V. M. L. L.; MATOS, F. R. N.; BASTOS, A. T. Administração contingencial em um centro de negócios: o caso do Beco da Poeira em Fortaleza - Ceará. In: XII SEMINÁRIOS EM ADMINISTRAÇÃO - SEMEAD, 2009, São Paulo. Anais... São Paulo, SP, 2009.

SILVA, E. S. da; SANTOS, M. C.; SILVA, J. B. da.
Comércio Informal no Centro de Fortaleza: Beco da Poeira e Feira da Sé. In: ENCONTRO NACIONAL DOS GEÓGRAFOS, 16, 2010, Porto Alegre. Anais... Porto Alegre, 2010. Disponível em: $\mathrm{h}$ ttp://www.agb.org.br/evento/ download. php?idTrabalho=2402>. Acesso em: 05 maio 2011.

STOKOLS, D. On the distinction between density and crowding: Some implications for future research, Psychological Review, n. 79, p. 275-279, 1972.

TELLER, C. Hedonic and utilitarian shopper types in evolved and created retail agglomerations. The International Review of Retail, Distribution and Consumer Research. v. 18, n. 3, p. 283-309, 2008.

TORRES, H.G.; BICHIR, R.M.; CARPIM, T.P. Uma pobreza diferente? Mudanças no padrão de consumo da população de baixa renda. Novos Estudos, Cebrap, n. 74, p. 17-22, 2006.

VELOSO, A. R.; HILDEBRAND, D. F. N.; DARE, P. R. C.; CAMPOMAR, M. C. A criança no varejo de baixa renda. RAE Eletrônica, v. 7, n. 2, p. 1-26, 2008. Disponível em: <http:// www.scielo.br/scielo.php?script $=$ sci_ arttext\&pid=S1676-56482008000200003>. Acesso em: 30 mai. 2012.

ZILBER, S. N.; SILVA, F. L. Estratégias das grandes empresas para o mercado brasileiro da base da pirâmide. Economia Global e Gestão, v.15, n.2, 2010. Disponível em: <http://www.scielo.oces. mctes.pt/pdf/egg/v15n2/v15n2a03.pdf>. Acesso em: 30 out. 2011. 